\title{
Physical Density Estimations of Single- and Dual- energy CT Using Material-based Forward Projection Algorithm
}

\section{Kai-wen Li}

Beihang University

\section{Daiyu FUjiwara}

Tokushima University

Akihiro Haga ( $\sim$ haga@tokushima-u.ac.jp )

Graduate School of Biomedical Sciences, Tokushima University, Tokushima 770-8503, Japan https://orcid.org/0000-0001-9220-2862

\section{Huisheng Liu}

Beihang University

\section{Li-Sheng Geng}

Beihang University

\section{Research}

Keywords: CT calibration, Dual-energy CT, Biological tissue, ICRP110 human phantom, HU-to-density curve

Posted Date: September 8th, 2020

DOl: https://doi.org/10.21203/rs.3.rs-67602/v1

License: (9) This work is licensed under a Creative Commons Attribution 4.0 International License. Read Full License 
1 Title: Physical density estimations of single- and dual-energy CT using material-based forward 2 projection algorithm

3 Running Title: Physical density estimations using material-based forward projection

4

5 Author:

6 Kai-Wen Li ${ }^{1,2}$, Daiyu Fujiwara ${ }^{3}$, Akihiro Haga ${ }^{3 a}$, Huisheng Liu ${ }^{1}$ and Li-Sheng Geng ${ }^{2,1,4,5}$

7 1. Beijing Advanced Innovation Center for Big Data-Based Precision Medicine, School of Medicine and Engineering, Beihang University, Key Laboratory of Big Data-Based Precision Medicine (Beihang University), Ministry of Industry and Information Technology, Beijing 100191, China

2. School of Physics, Beihang University, Beijing 102206, China

3. Graduate School of Biomedical Sciences, Tokushima University, Tokushima 770-8503, Japan

4. Beijing Key Laboratory of Advanced Nuclear Materials and Physics, Beihang University, Beijing 102206, China

5. School of Physics and Microelectronics, Zhengzhou University, Zhengzhou, Henan 450001, China

\section{Corresponding Author:}

Akihiro Haga, Ph.D.

Electronic mail: haga@tokushima-u.ac.jp

19 Telephone number: +81886339024

20 Mailing address: 3-18-15 Kuramoto-cho, Tokushima, 770-8503, Japan 
Abstract

Purpose: This study aims to evaluate the accuracy of physical density prediction in single-energy CT (SECT) and dual-energy CT (DECT) by adapting a fully simulation-based method using a materialbased forward projection algorithm (MBFPA).

Methods: We used biological tissues referenced in ICRU Report 44 and tissue substitutes to prepare three different types of phantoms for calibrating the HU-to-density curves. Sinograms were first virtually generated by the MBFPA with four representative energy spectra (i.e. $80 \mathrm{kV}, 100 \mathrm{kV}, 120 \mathrm{kV}$, and $6 \mathrm{MV}$ ) and then reconstructed to form realistic CT images by adding statistical noise. The HU-todensity curves in each spectrum and their pairwise combinations were derived from the CT images. The accuracy of these curves was validated using the ICRP110 human phantoms.

Results: The relative mean square errors (RMSEs) of the physical density by the HU-to-density curves calibrated with $\mathrm{kV}$ SECT nearly presented no phantom size dependence. The $\mathrm{kV}-\mathrm{kV}$ DECT calibrated processes that control the variance of patient dose calculations in radiotherapy treatment planning ${ }^{1-3}$. curves were also comparable with those from the $\mathrm{kV}$ SECT. The phantom size effect became notable when the MV X-ray beams were employed for both SECT and DECT due to beam hardening effects. The RMSEs were decreased using the biological tissue phantom.

Conclusions: Simulation-based density prediction can be useful in the theoretical analysis of SECT and DECT calibrations. The results of this study indicated that the accuracy of SECT calibration is comparable with that of DECT using biological tissues. The size and shape of the calibration phantom could affect the accuracy, especially for MV CT calibrations.

Keywords: CT calibration, Dual-energy CT, Biological tissue, ICRP110 human phantom, HU-todensity curve

\section{Introduction}

To establish the relationship between the computed tomography (CT) number (in Hounsfield units, $\mathrm{HU}$ ) of a given voxel and the physical (or electron) density relative to water is one of the crucial The HU-to-density conversion is typically determined by calibration curves, which are experimentally 
obtained from tissue-substitutes with known densities in a calibration phantom. Several studies have investigated the sensitivity of dose calculation for photon and particle beams relative to the accuracy of this conversion ${ }^{4-7}$. A major concern of this approach is that the elemental composition of these substitutes may differ from that of biological tissues, and consequently, the adopted calibration curves may not be sufficiently accurate. One way to overcome this problem is the state-of-the-art stoichiometric calibration method introduced by Schneider et al. ${ }^{8}$, in which the specific parameters of a CT scanner are determined by the measurement of a few tissue-substitutes with known materials. Recently, this method has been re-examined in the context of single-energy CT (SECT) calibration for proton therapy treatment planning, and its accuracy was found to depend on the tissue-substitutes used for calibration ${ }^{9}$. This dependency implies that the improvement of dual energy CT (DECT) over SECT should also be reassessed, which may depend on the use of tissue substitutes as determined in a number of experiments for predicting relative stopping powers in proton therapy ${ }^{10}$.

In this study, the accuracy of physical density prediction using SECT and DECT was evaluated, which is the first step in treatment planning. To reduce the uncertainty of the calibration, the HU-to-density conversion using biological tissues from ICRU Publication 44 was proposed ${ }^{11-15}$. The approach in this study differs from the stoichiometric calibration ${ }^{8}$ by assuming that the X-ray spectra are known for the evaluation of the attenuation coefficients. In this case, CT values were reconstructed from the sinograms generated by the material-based forward projection algorithm (MBFPA), which has been utilised in model-based material decomposition ${ }^{16,17}$, when the material composition of the object was determined. These CT values were then used to perform the calibration to obtain the HU-to-density look-up-table (LUT). Using this LUT, arbitrary CT images can be converted to density images. Namely, this approach is a full simulation of the clinical process because it is based on the modelling of the entire CT system, including the incident X-ray energy spectrum.

The aim of this study was threefold. First, the feasibility of the proposed approach using MBFPA for physical density prediction was presented, which is significantly relevant to estimate proton stopping powers for treatment planning. To achieve this, the HU-to-density curves were simulated using three types of phantoms, where phantom (size/shape and composition) dependence in the calibration was also analysed. Second, the advantage of DECT over SECT for calibrations based on the proposed approach 
was quantitatively evaluated. Furthermore, the issue of whether a large energy gap in DECT, such as in the kilovoltage-megavoltage (kV-MV) range, could improve the accuracy of estimating the physical density was reassessed.

\section{Materials and Methods}

81 The schematic workflow is shown in Fig. 1. The method starts with the preparation of three types of 82 two-dimensional material-based digital calibration phantoms. Next, virtual projections (or sinograms) 83 were produced using MBFPA, and CT images were sequentially reconstructed with the sinograms. 84 Using the reconstructed images, the HU-to-density LUTs were calculated for each phantom. Finally, these LUTs were validated by predicting the physical density distributions of the ICRP110 human phantom. Four different X-ray energy sources (i.e. 80 kV, 100 kV, 120 kV, $6 \mathrm{MV}$ ) were employed. Thus, four SECTs and their six pairwise combinations for DECTs were considered.

\section{Calibrations}

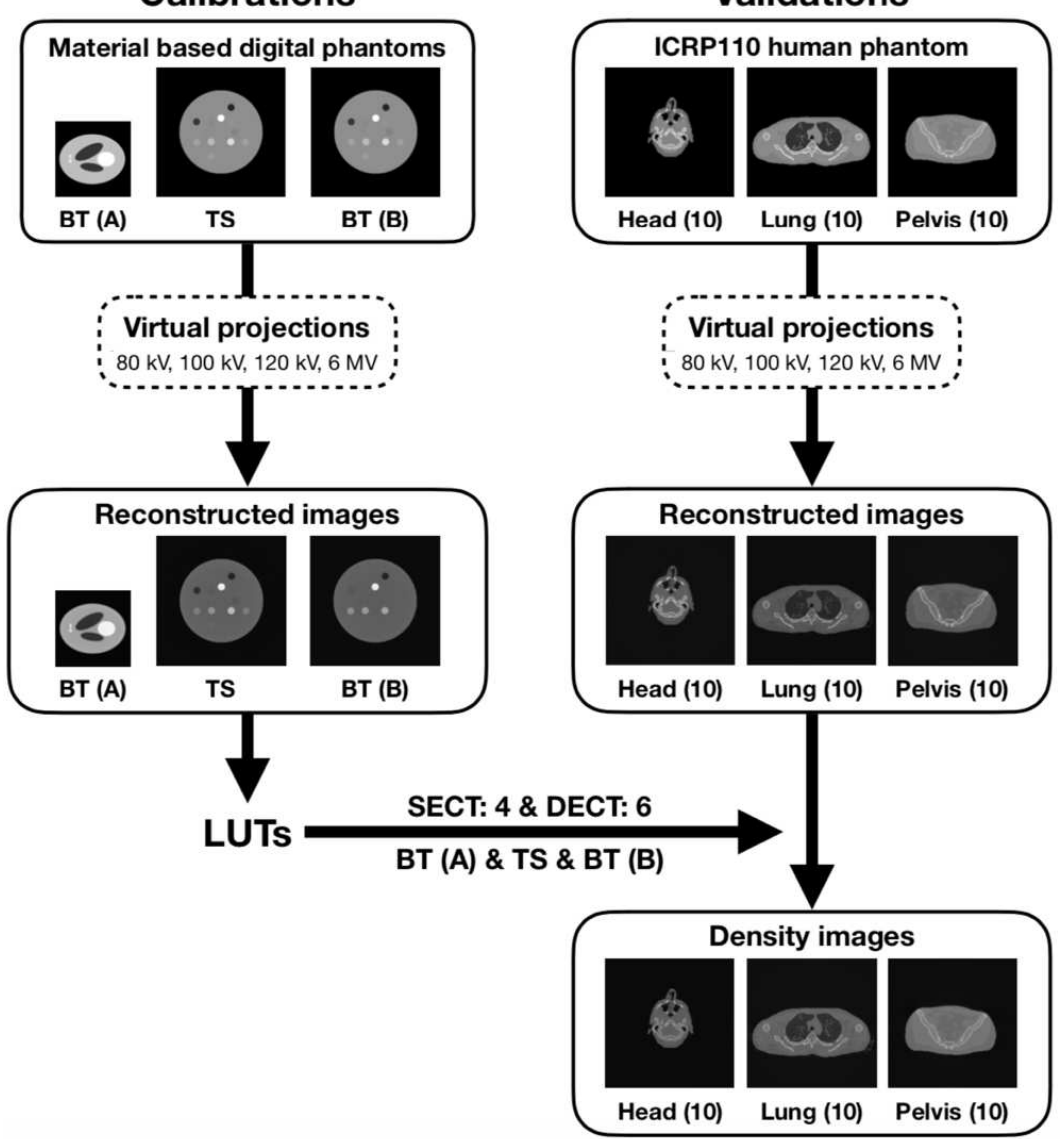

Fig. 1. Workflow of the current study for total density evaluation. BT(A), TS, and BT(B) phantoms were used for the calibration, and the ICRU human phantoms were used for the validation (for more details, see the main text). 
91 Three types of phantoms were employed for the calibrations in this study, including the biological tissue (BT) phantoms in two different sizes and shapes, as well as the Gammex phantom (Gammex Inc., Middleton, WI). The former two are referred to as the BT(A) and BT(B) phantoms, respectively. The latter is known to have better tissue substitutes (TS) than the other tissue-mimicking phantoms ${ }^{9}$, and is hereon referred to as the TS phantom. The shape of BT(A) is similar to the Shepp-Logan phantom, whereas those of TS and BT(B) mimicked that of the Gammex phantom. The composed materials of BT(A) were taken from the ICRU publication $44^{13-15}$. Details of the elemental compositions and mass fractions are listed in Table I of Ref. 11 or Table I of Ref. 12. Following Hünemohr et al.'s study ${ }^{12}$, the mass fractions of six major elements $(\mathrm{H}, \mathrm{C}, \mathrm{N}, \mathrm{O}, \mathrm{P}, \mathrm{Ca})$ were normalised to provide a sum of $100 \%$. Three of the materials were randomly selected to construct one BT(A) phantom; a total of 54 phantoms were generated. Meanwhile, the TS phantom was composed of one base material (solid water) and 12 insertions (LN-300, LN-450, AP6, BR12, water, SR2, LV1, IB3, B200, CB2-30\%CaCO, CB2$50 \% \mathrm{CaCO}_{3}$, and $\left.\mathrm{SB} 3\right)$, which were generated with weight fractions and mass densities of 8 elements $(\mathrm{H}$, $\mathrm{C}, \mathrm{N}, \mathrm{O}, \mathrm{Mg}, \mathrm{Si}, \mathrm{P}$, and $\mathrm{Ca}$ ) provided in Ref. ${ }^{9}$. The $\mathrm{BT}(\mathrm{B})$ phantoms have the same shape as the TS phantom but consist of 12 standard human biological tissues ${ }^{13-15}$ and water instead of tissue-substitutes. By using different biological tissues, 6 phantoms were created. The width (major axis) and height (minor axis) of each BT(A) phantom were $260 \mathrm{~mm}$ and $197 \mathrm{~mm}$, respectively, with a pixel scale of $1 \mathrm{~mm}$, while the TS and BT(B) phantoms had a radius of $115 \mathrm{~mm}$. Each of the insertions in the latter two had a radius of $13 \mathrm{~mm}$. All the phantoms were placed in the air to obtain "complete" phantoms (images), for which the sizes were $300 \times 300 \mathrm{~mm}^{2}$ for the BT(A) and $512 \times 512 \mathrm{~mm}^{2}$ for the TS and BT(B). These three types of phantoms were considered for the following reasons: 1) to analyse the size and shape dependence by comparing the calibration results from the BT(A) and BT(B) phantoms, and 2) to analyse the material dependence by comparing the TS and BT(B) phantoms. Finally, 30 images were prepared

114 from the ICRP110 human phantoms for validation, which consisted of five head, lung, and pelvis 115 phantom slices for both, females and males, which were all $512 \times 512 \mathrm{~mm}^{2}$ in size with a 1-mm scale; 116 six elements $(\mathrm{H}, \mathrm{C}, \mathrm{N}, \mathrm{O}, \mathrm{P}$, and $\mathrm{Ca})$ were considered. The specifics of all the phantoms used in this 117 study are summarised in Table 1. The phantoms in the calibration are shown in Fig. 2, and the selected 


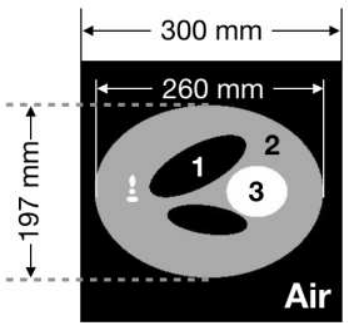

BT(A)

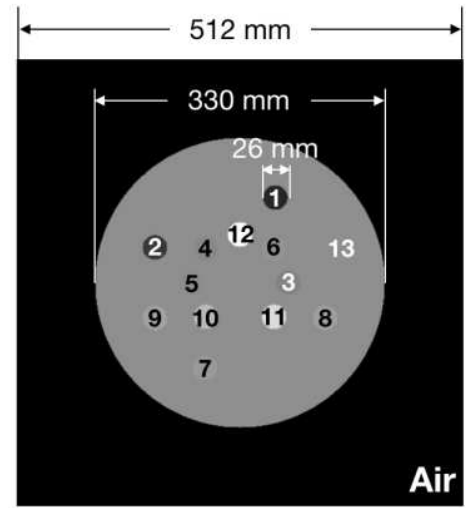

TS

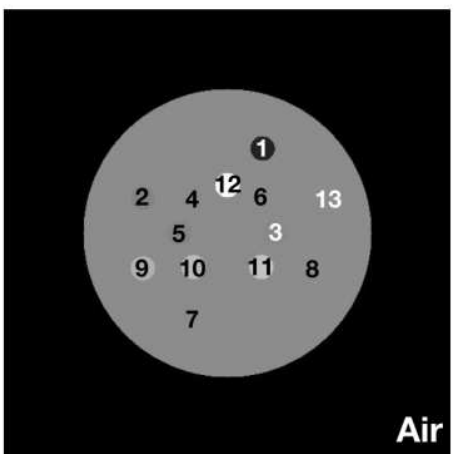

BT(B)

- BT(A) 1-3: biological tissues

- TS 1: LN-300, 2: LN-450, 3: AP6, 4: BR12, 5: Water, 6: SR2, 7: LV1, 8: IB3, 9: B200, 10: $\mathrm{CB} 2-30 \% \mathrm{CaCO}$, 11: $\mathrm{CB} 2-50 \% \mathrm{CaCO}$, 12: SB3, 13: Solid water

- BT(B) 5: Water, Others: biological tissues

Fig. 2. Shape, size, and composition of the BT(A), TS, and BT(B) phantoms in the calibration.
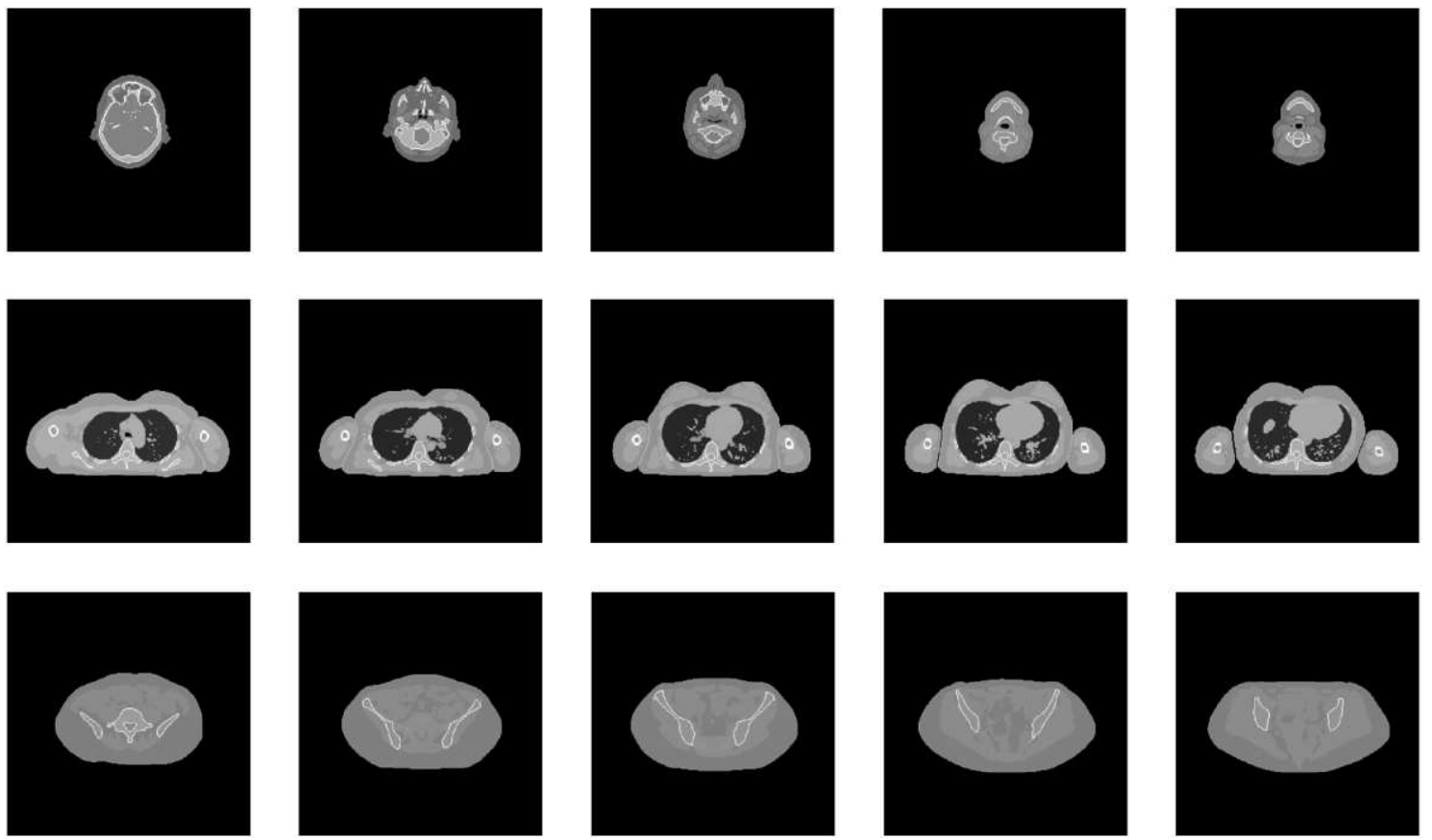

Fig. 3. Ground truth of the selected ICRP110 human phantoms for female. The images in the first, second, and third row indicate head, lung, and pelvis, respectively. 

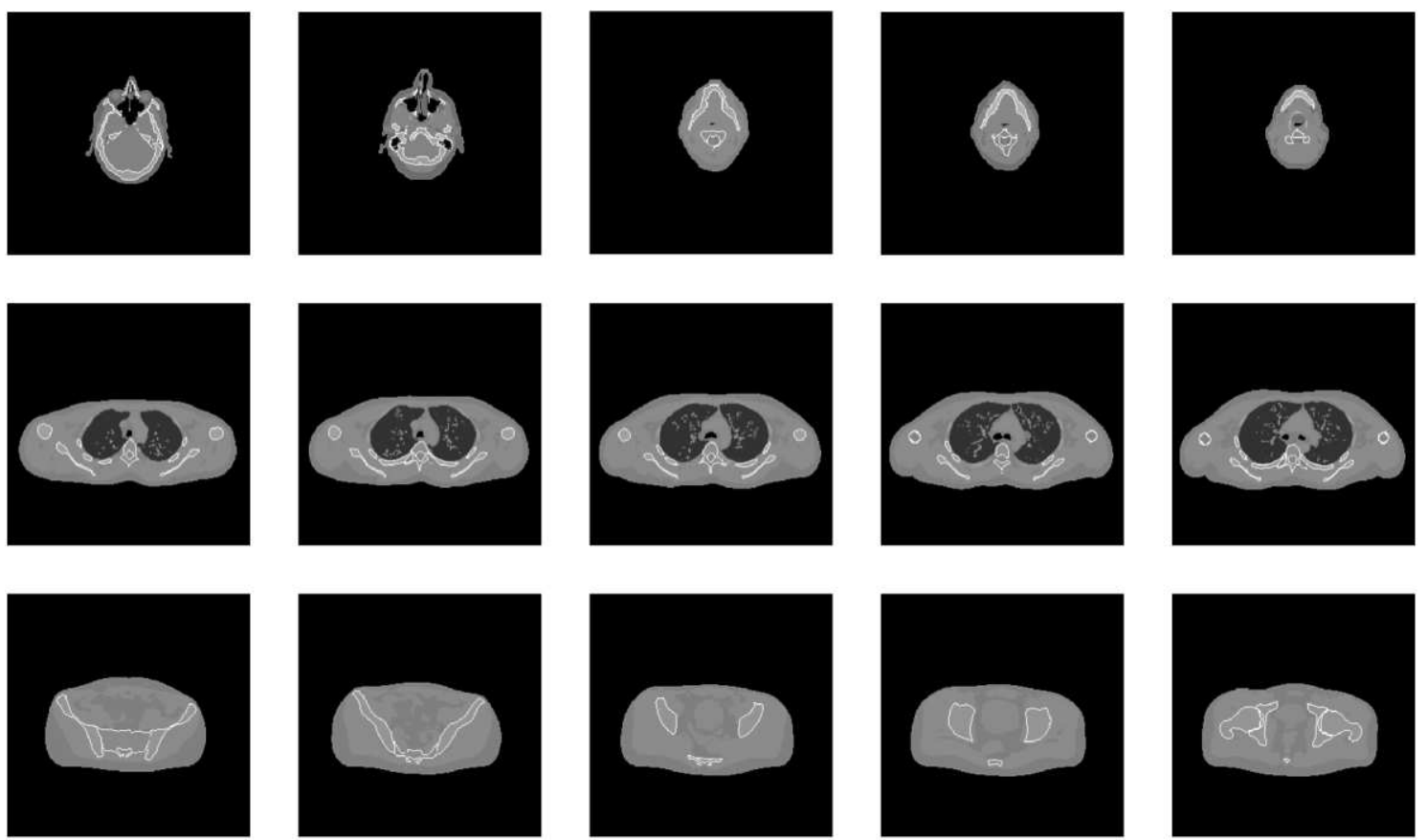

Fig. 4. Ground truth of the selected ICRP110 human phantoms for male. The images in the first, second, and third row indicate head, lung, and pelvis, respectively.

Table 1. Specifics of all the phantoms used in this study.

\begin{tabular}{lccccc}
\hline & \multicolumn{3}{c}{ Calibration } & & Validation \\
\cline { 2 - 4 } Name & BT (A) & TS & BT (B) & & ICRP110 human \\
\hline Materials & Biological tissues & Tissue substitutes & Biological tissues & & - \\
Shape & Shepp-Logan & Gammex & Gammex & & Head/Lung/Pelvis \\
Width (mm) & 260 & 330 & 330 & & - \\
Height $(\mathrm{mm})$ & 197 & 330 & 330 & - \\
Image $\left(\mathrm{mm}^{2}\right)$ & $300^{2}$ & $512^{2}$ & $512^{2}$ & \\
Slice $(\mathrm{s})^{\dagger}$ & 54 & 1 & 6 & $312^{2}$ \\
\hline \hline
\end{tabular}

$\uparrow:$ number of phantoms (slices) used in calibration and validation.

\section{Virtual projection and reconstruction}

132 In this study, four different X-ray energies (80 kV, $100 \mathrm{kV}, 120 \mathrm{kV}$, and $6 \mathrm{MV})$ were used for SECTs,

133 and their six pairwise combinations for DECTs. This makes it simple to compare the results from SECT,

$134 \mathrm{kV}-\mathrm{kV}$ DECT, and kV-MV DECT. For diagnostic CT, an X-ray within the MV range is not applicable,

135 whereas for image-guided radiation systems, equipment to produce clinical or non-clinical projection

136 images with MV X-rays exists ${ }^{18-20}$. In this study, a 6 MV X-ray, which is a typical X-ray energy used in 
radiation treatment, was selected. The spectra of the X-ray sources were obtained by Monte-Carlo (MC)

138 simulations using the GEANT4 toolkit (version 10.4) for a linear accelerator with kV imaging capability

139 (Synergy, Elekta, UK). For kV X-rays, low-energy photons generated from an anode were decimated

140 by filters composed of aluminium and copper, and the beam shape was formed by lead-cone and cassette

141 collimators. For the MV X-rays, the photons generated from the target were decimated by a flattening

142 filter, and the beam shape was formed by primary, jaw, and multi-leaf collimators. In both cases, the

143 energy spectrum was formed by the photons collected on the plane located $70 \mathrm{~cm}$ from the sources ${ }^{21}$.

144 Fig. 5 presents the X-ray spectra produced for the $\mathrm{kV}$ and MV beams. Using the spectra and the digital

145 calibration phantoms indicated above, virtual projections using MBFPA were then simulated; further

146 details can be found in the Appendix. The geometry of the simulated CTs with relevant factors is shown

147 in Fig. 6. The source and detectors were rotated 360 degrees in 0.45 -degree increments (in total 800

148 projections). There were 609 detectors, which were aligned in equal intervals $(0.15 \mathrm{~cm})$. Furthermore,

149 random noise is inevitable in CT imaging, which is also a main source of artefacts. A Gaussian

150 distributed random noise was added to the sinograms (detectors) for the simulations in this study, for

151 which the strength was determined to produce a signal-to-noise ratio of $\sim 20$ in the reconstructed images

152 of a homogeneous water phantom, which is consistent with that in clinical practice.
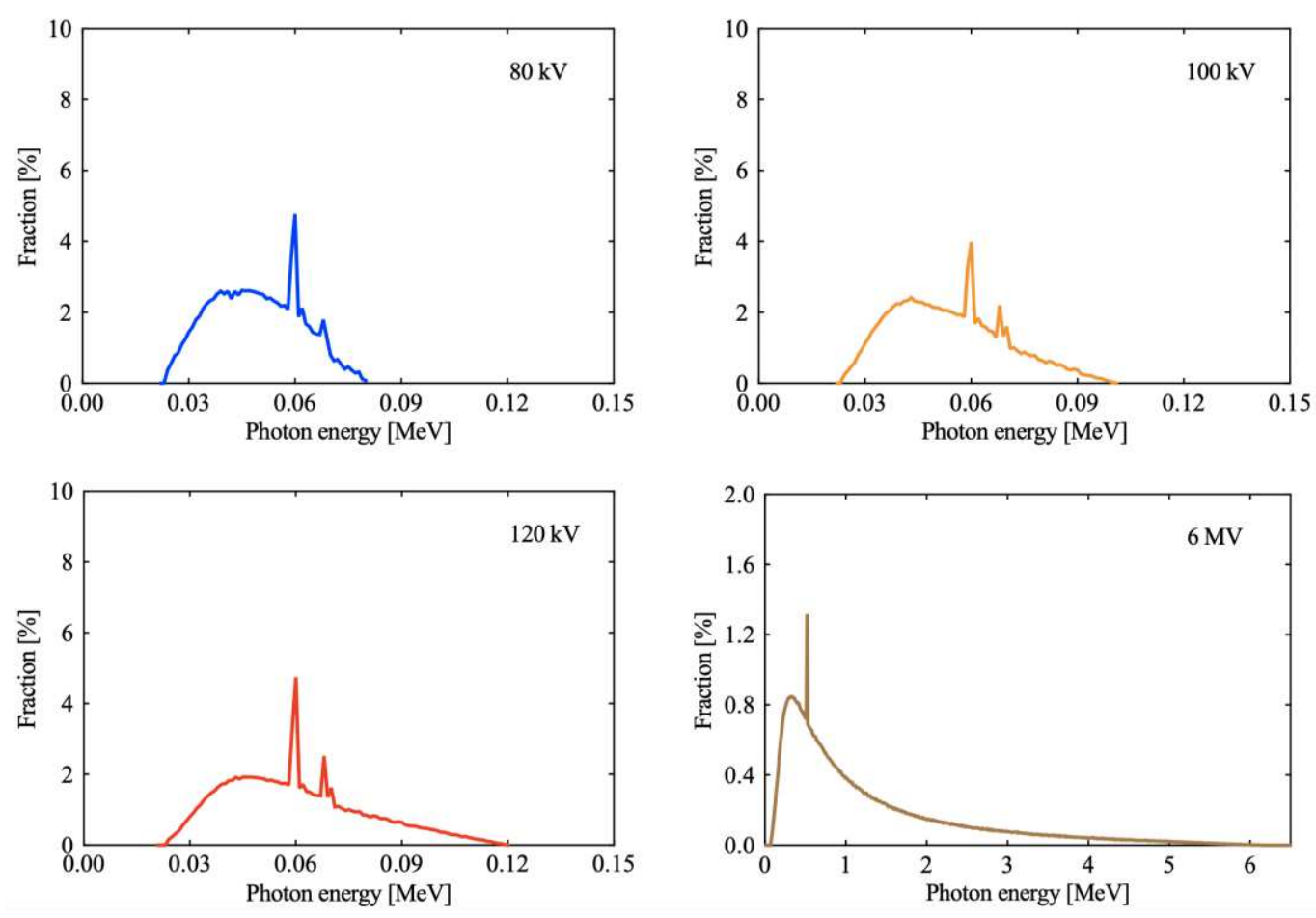

153

Fig. 5. Simulated X-ray spectra of 80 kV, 100 kV, 120 kV, and $6 \mathrm{MV}$. The mean energies were $48.70 \mathrm{keV}$, $53.24 \mathrm{keV}, 59.28 \mathrm{keV}$, and $1.30 \mathrm{MeV}$, respectively. 


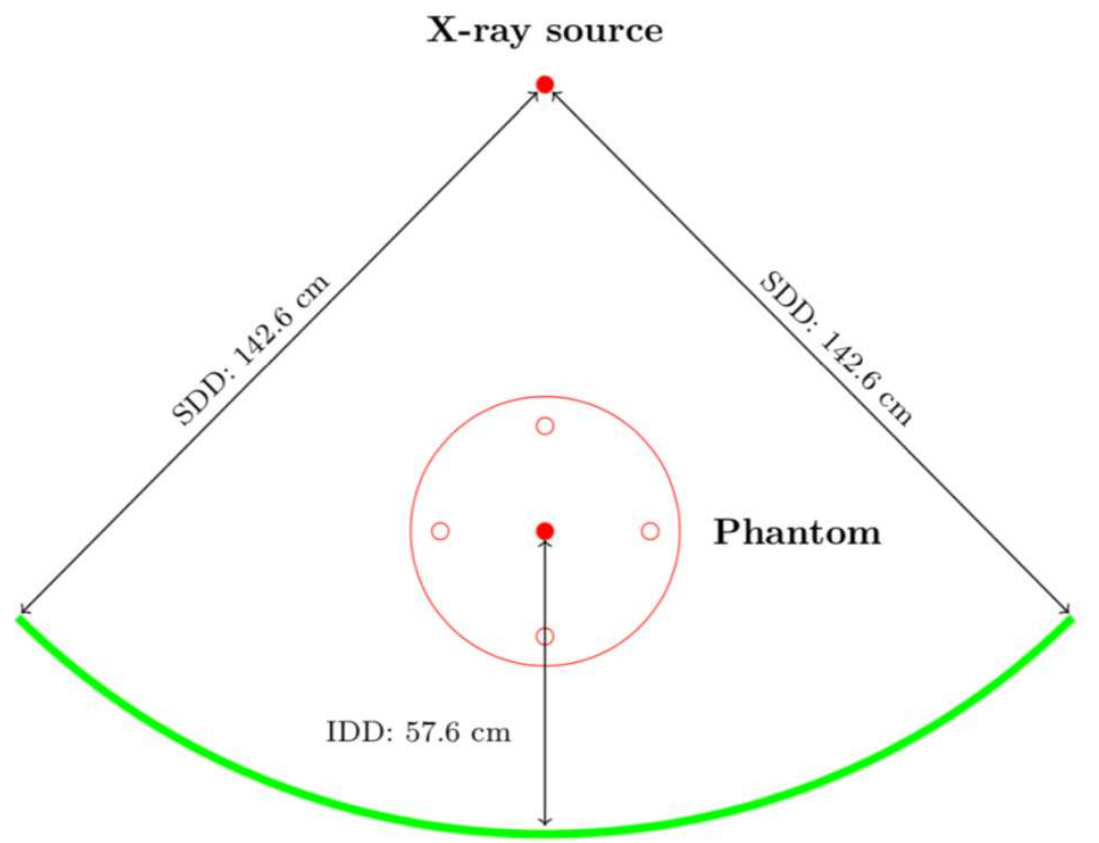

Vitrual detectors

Fig. 6. Schematic of geometry applied in sinogram production. IDD and SDD indicate the isocenter-todetector distance and the source-to-detector distance, respectively. A total of 609 detectors were used, and the size of each one was $0.15 \mathrm{~cm}$.

\section{HU-to-density relation for SECT calibrations}

160 For the calibrations, the average CT number (HU) of each material from the reconstructed images

161 along with the corresponding physical density (known for the calibration phantoms) were used to form

162 the LUTs. A fourth order polynomial was applied to relate the physical density $\rho$ with HU in

163 Hounsfield units as follows:

$$
\rho(H U)=c_{0}+c_{1} H U+c_{2} H U^{2}+c_{3} H U^{3}+c_{4} H U^{4},
$$

where $c_{i}(i=0, \cdots, 4)$ are the parameters; note, $c_{0}$ can be expressed with $c_{i}(i=1, \cdots, 4)$ calibration phantoms and should be treated accordingly.

170 The $\Delta H U-\rho_{e}$ conversion method ${ }^{22,23}$ was applied for the DECT calibration, however, the electron

171 density $\rho_{e}$ was replaced with the physical density $\rho$. This is appropriate because $\rho$ is proportional to 
$\rho_{e}$. The dual energy subtracted quantity $\Delta H U$ was defined as follows:

$$
\Delta H U \equiv(1+\alpha) \mathrm{HU}_{H}-\alpha \mathrm{HU}_{L},
$$

where $\mathrm{HU}_{H}$ and $\mathrm{HU}_{L}$ denote the high-energy and low-energy $\mathrm{CT}$ numbers in Hounsfield units, respectively. Further, $\alpha$ is a weighting factor for the subtraction, which is regarded as materialindependent. Similar to a previous study ${ }^{22}$, the relation between $\Delta H U$ and $\rho$ was assumed to be linear for materials with low effective atomic numbers as follows:

$$
\rho(\Delta H U)=a \frac{\Delta H U}{1000}+b
$$

where $a, b$, and $\alpha$ can be determined by a least squares fit to $\left(\mathrm{HU}_{H}, \mathrm{HU}_{L}\right)-\rho$ data obtained from DECT scans of materials with a known density $\rho$ in calibration phantoms, which is similar to the SECT calibration.

\section{Validation}

The minimum $\chi^{2}$ value of the fitting curves for the physical density were evaluated for all three calibration phantoms. A total of 30 virtual images based on the ICRP110 human phantoms (shown in Table I and Figs. 3 and 4) were independently prepared via virtual projections and image reconstructions. The physical density distribution converted by the LUTs of each energy spectrum and calibration phantom were compared with the ground truth. Statistical analysis was performed to determine the differences in the RMSE among the chosen energies (for SECT), their combinations (for DECT), or the chosen calibration phantoms. In particular, the following differences were assessed: 1) between SECT and DECT, 2) between TS and BT(B), and 3) among the energies with BT(A) and BT(B). For the statistical analysis, a Student's t-test was employed for the first two cases, while Tukey's range test was employed for the last.

\section{Results}

\section{Generated sinograms and reconstructed images}

Fig. 7 presents the representative sinograms generated from the MBFPA of the TS phantom with four energies, and Fig. 8 presents the corresponding reconstructed images. 

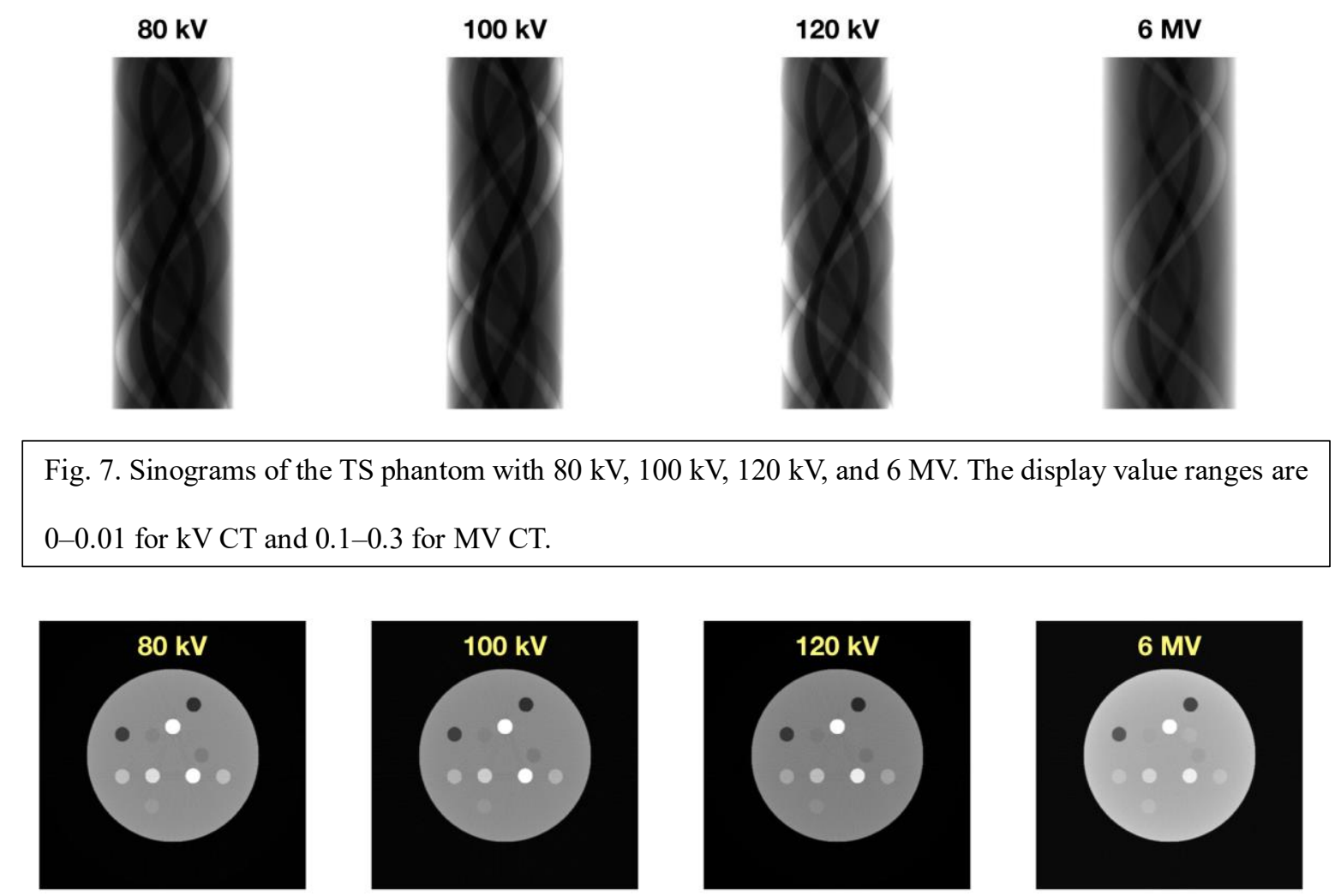

Fig. 8. Reconstructed images of the TS phantom with $80 \mathrm{kV}, 100 \mathrm{kV}, 120 \mathrm{kV}$, and $6 \mathrm{MV}$. The display value ranges of the attenuation coefficients (in units of $\mathrm{cm}-1$ ) are $0-0.4$ for $\mathrm{kV} \mathrm{CT}$ and $0-0.1$ for MV CT.

\section{SECT calibration results}

208 A dataset of CT values and physical densities in the calibration phantoms can be derived from the 209 above reconstructed images. The data points and fitted curves for all three phantoms and four energies

210 are shown in Fig. 9. The three calibration phantoms present similar behaviours, which is reasonable 211 because similar or same materials were used. However, the BT(A) phantoms present a relatively 212 different behaviour compared to the TS and BT(B) phantoms in the case of 6 MV because the size of 213 the former is smaller. 

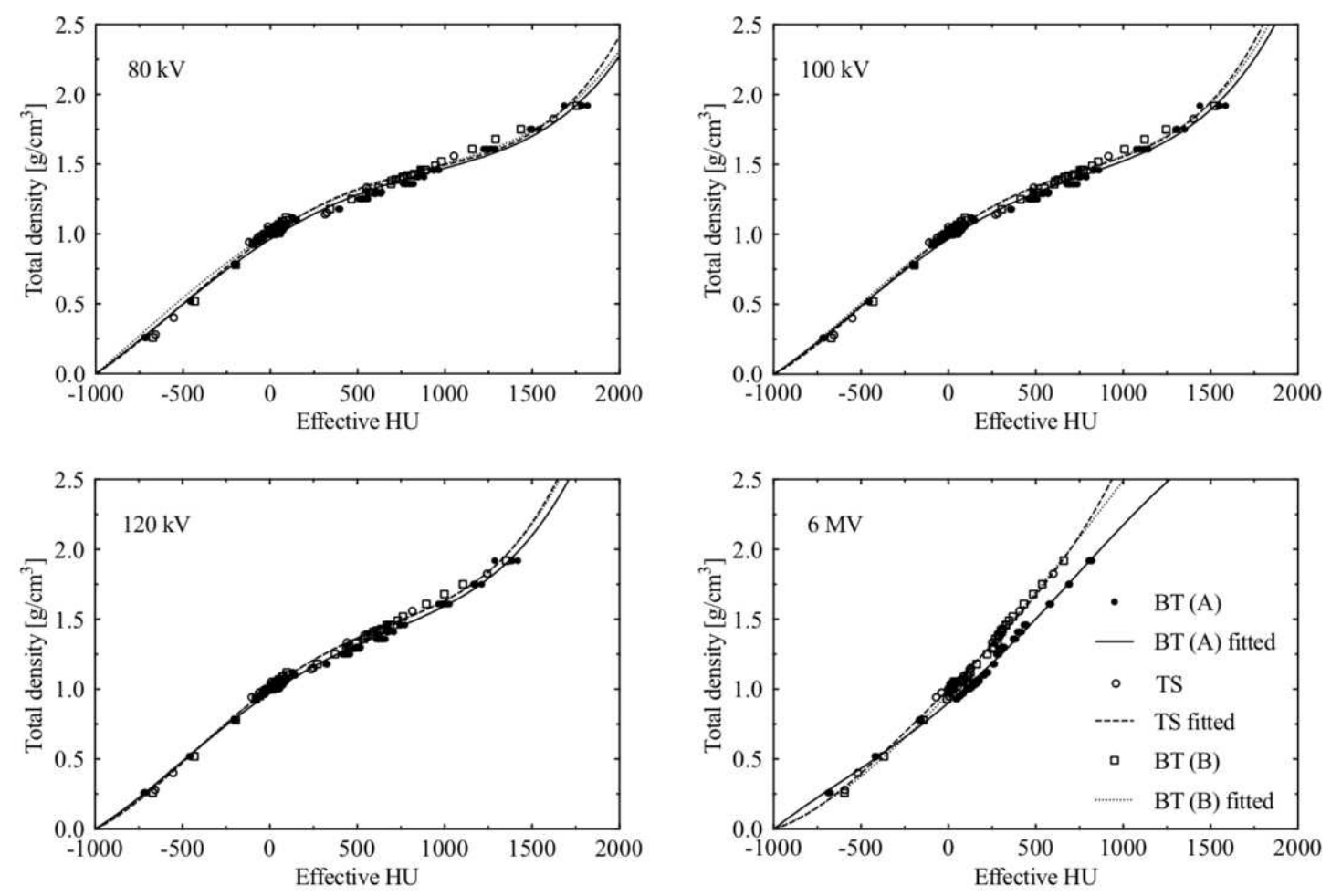

Fig. 9. HU-to-density relations of three calibration phantoms with $80 \mathrm{kV}, 100 \mathrm{kV}, 120 \mathrm{kV}$, and $6 \mathrm{MV}$ in SECT. The CT values are referred to as "Effective HU" because this approach is based on simulations.

\section{DECT calibration results}

The data of $\Delta H U$ and $\rho$, as well as the fitted results, are shown in Fig. 10. It was found that the TS and BT(B) phantoms have similar behaviours in all six DECT calibrations, which are different from the BT(A) phantoms. However, in the case of $\mathrm{kV}$ SECTs, all three curves present similar behaviours, as shown in Fig. 9. This is because a fourth order polynomial was applied for the SECT fitting, while a linear relationship was assumed for the DECT calibration. Therefore, the difference in the data points is suppressed by the higher order terms in the SECT situation. However, for the DECT calibration, such differences become notable. In addition, the fact that the size/shape of BT(A) and BT(B) phantoms are different also implies that the physical density estimation in the $\Delta H U$ approach depends on (the size/shape of) the calibration phantoms as well. In particular, this dependence appears stronger in $\mathrm{kV}$ MV DECT calibrations. 

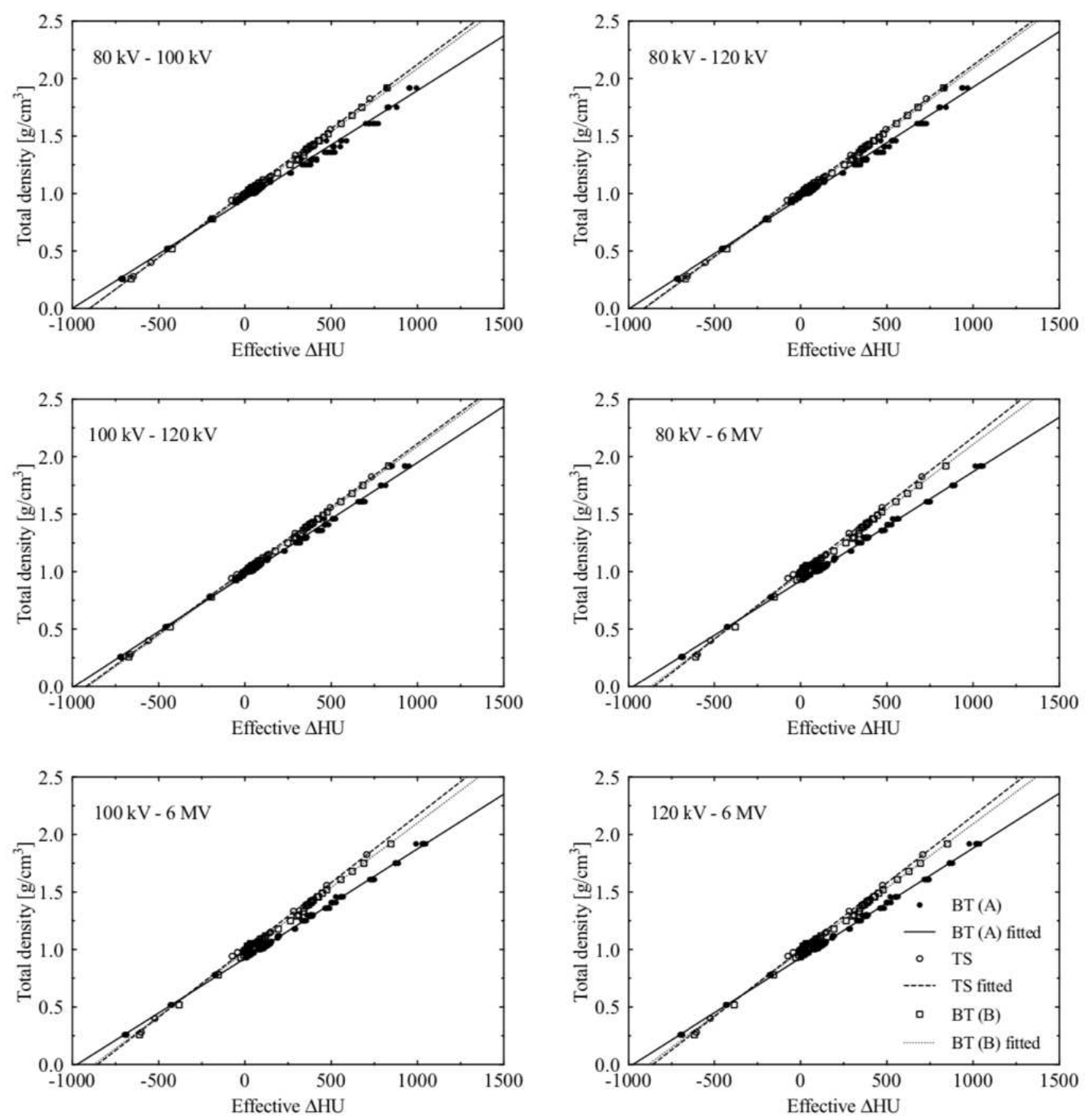

Fig. 10. Effective $\Delta \mathrm{HU}$ to density relations of the three calibration phantoms with $80 \mathrm{kV}, 100 \mathrm{kV}, 120 \mathrm{kV}$, and $6 \mathrm{MV}$ in DECT.

\section{Validation using the ICRP110 human phantom}

238 The validation results using the ICRP110 human phantom are shown in Table II, where the average 239 RMSEs are listed for all the anatomies. No significant difference in the RMSEs was found between 240 SECT and DECT ( $p$-values: 0.26, 0.16, and 0.68 for BT(A), TS, and BT(B), respectively). Conversely,

241 the predicted results from the calibrations using BT(B) phantom significantly improved compared to 242 that using TS phantom in density calibration ( $p$-values: $<0.01$ ), although the mean RSME difference 243 was less than 0.0093. For the energy dependence of the physical density estimation, the RMSEs of the 
2446 MV SECT were observed to be significantly larger than those of the others for BT(B), whereas no

245 significant difference was observed in the use of BT(A), mainly caused by the lung area having the large 246 size. In addition, the RMSEs of the 6 MV SECT from BT(A) phantoms are relatively better than those 247 from BT(B) phantoms. More specifically, the smaller the calibration phantom, the more accurate the 248 validation predictions are. However, such behaviours are not apparent in the kV SECT results.

250 Table 2. Predicted average RMSEs of ICRP110 phantom slices from BT(A), TS, and BT(B) phantoms with SECT 251 and DECT.

\begin{tabular}{|c|c|c|c|c|c|c|c|c|c|c|c|}
\hline \multirow[b]{2}{*}{ Calibration } & \multirow[b]{2}{*}{ Anatomy } & \multicolumn{4}{|c|}{ SECT predictions } & \multicolumn{6}{|c|}{ DECT predictions } \\
\hline & & $80 \mathrm{kV}$ & $100 \mathrm{kV}$ & $120 \mathrm{kV}$ & $6 \mathrm{MV}$ & $80 \mathrm{kV}-100 \mathrm{kV}$ & $80 \mathrm{kV}-120 \mathrm{kV}$ & $100 \mathrm{kV}-120 \mathrm{kV}$ & $80 \mathrm{kV}-6 \mathrm{MV}$ & $100 \mathrm{kV}-6 \mathrm{MV}$ & $120 \mathrm{kV}-6 \mathrm{MV}$ \\
\hline \multirow{3}{*}{ BT(A) } & Head & 0.1230 & 0.1236 & 0.1197 & 0.1270 & 0.1264 & 0.1180 & 0.1128 & 0.1155 & 0.1160 & 0.1156 \\
\hline & Lung & 0.1507 & 0.1429 & 0.1356 & 0.0978 & 0.1548 & 0.1394 & 0.1516 & 0.0950 & 0.0963 & 0.0978 \\
\hline & Pelvis & 0.0793 & 0.0756 & 0.0739 & 0.0750 & 0.0793 & 0.0781 & 0.0785 & 0.0759 & 0.0754 & 0.0750 \\
\hline \multirow{3}{*}{ TS } & Head & 0.1362 & 0.1424 & 0.1395 & 0.2289 & 0.1758 & 0.1542 & 0.1395 & 0.1981 & 0.1967 & 0.1934 \\
\hline & Lung & 0.1589 & 0.1522 & 0.1445 & 0.1237 & 0.1873 & 0.1595 & 0.1728 & 0.1144 & 0.1141 & 0.1135 \\
\hline & Pelvis & 0.0759 & 0.0762 & 0.0770 & 0.1267 & 0.0951 & 0.0917 & 0.0903 & 0.1121 & 0.1108 & 0.1089 \\
\hline \multirow{3}{*}{$\mathrm{BT}(\mathrm{B})$} & Head & 0.1294 & 0.1375 & 0.1368 & 0.2217 & 0.1644 & 0.1454 & 0.1327 & 0.1771 & 0.1749 & 0.1702 \\
\hline & Lung & 0.1484 & 0.1456 & 0.1418 & 0.1172 & 0.1809 & 0.1558 & 0.1687 & 0.1046 & 0.1048 & 0.1050 \\
\hline & Pelvis & 0.0727 & 0.0745 & 0.0757 & 0.1144 & 0.0876 & 0.0850 & 0.0842 & 0.0934 & 0.0918 & 0.0896 \\
\hline
\end{tabular}

\section{Discussions}

254 This study presented the evaluation of both SECT and DECT calibrations based on the MBFPA and 255 demonstrated that the accuracy of SECT calibration is comparable with that of DECT. In addition, the 256 use of the biological tissue phantom in the calibration was found to improve the physical density 257 prediction compared with that of the Gammex phantom composed of tissue substitutes.

258 Similar results have already been implicitly obtained by Goma et al. who performed SECT calibration 259 using the stoichiometric method, which consists of characterising the CT scanner directly through CT 260 scans of tissue substitutes, and subsequently predicting the CT numbers of biological tissues using this 261 characterisation ${ }^{9}$. The main difference between the stoichiometric calibration and the present MBFPA 262 approach is that the former requires real CT images acquired from CT scanners, whereas the latter does 
not. In this study, the CT scanners were modelled, characterised by X-ray energy spectra directly, by which various simulations, with not only the $\mathrm{kV-range} \mathrm{X-rays} \mathrm{but} \mathrm{also} \mathrm{MV-range} \mathrm{X-rays,} \mathrm{could} \mathrm{be}$ performed. Furthermore, the density results cannot be validated for real patients in the stoichiometric calibration framework. However, using the proposed MBFPA-based calibration approach, the validation is now possible by preparing, for example, the reconstructed CT datasets using the ICRP110 human phantom, which could be considered as real patients to a certain extent. This study not only supported the results of Goma et al. ${ }^{9}$ but also newly presented that the tissue substitutes differ from the biological tissues in physical density calibration.

271 The results of this study indicated that kV-MV DECT is not as outperformed as it was expected to be. This is due to the large beam hardening effect in MV CT, compared to that in $\mathrm{kVCT}$. This can be inferred 273 from the fact that different sizes of the calibration phantoms provided different calibration curves with MV X-rays. For a more apparent indication, a simulation using monochromatic energy X-rays with 3 $\mathrm{MeV}$ was also performed, which does not suffer from beam hardening. In this case, no phantom size dependence in the calibration curves was observed. The magnitude of beam hardening in MV CT could also be observed in the homogeneous water phantom (of the same size as the TS phantom) by extracting the reconstructed $\mathrm{CT}$ values in the centre and peripheral regions. Their relative difference was $\sim 17 \%$ for MV CT due to the cupping artefacts; however, this value is only $\sim 10 \%$ for $\mathrm{kV} \mathrm{CT}$. Such ambiguity in 280 the MV CT with a practical spectrum significantly affected the accuracy of the calibration. Hence, DECT 281 with MV X-rays does not improve the accuracy of the physical density estimation as well as the SECT 282 of MV X-rays. Furthermore, the use of MV X-rays passing through a titanium filter could, to a certain 283 extent, reduce the beam-hardening effect.

284 Note that Yang et al. assessed the superiority of kV-MV DECT in determining proton stopping power 285 by generating $1 \mathrm{MV}$ beams from MC calculations ${ }^{24}$. According to the authors, when CT number 286 uncertainties and artefacts such as imaging noise and beam hardening effects were considered, the $k \mathrm{~V}$ -

287 MVDECT improved the perfectly of SPR estimation substantially over kV-kV or MV-MVDECT methods.

288 The SPR estimation is directly influenced by the electron density (or physical density), and therefore, 289 Yang et al.'s study implied a substantial decrease in the physical density uncertainties in kV-MV DECT. 290 However, this study apparently supports the contrary. This might indicate that the beam hardening 
291 effects were underestimated in MV CT in Yang et al.'s study because the authors assumed the "average

292 spectra" accurately modelled the CT scanner. Thus, the beam hardening effect should be carefully 293 treated in MV CT.

294 The proposed method can be considered an improvement over previous stoichiometric approaches in 295 which the parameters, depending on the X-ray spectrum, which characterise the CT scanner are 296 determined by fitting to the effective linear attenuation coefficients of a given material, whereas the 297 proposed method explicitly deals with the X-ray spectrum. Although the explicit handling of the X-ray 298 spectrum is advantageous in CT calibration, the requirement of the X-ray spectrum imposes limitations 299 for practical applications. That is, the exact energy spectrum of medical CT scanners is unknown, and 300 its direct measurement is difficult because of the high photon flux. Nevertheless, novel methods to 301 estimate X-ray spectra in practical CT scanners have been proposed in recent years ${ }^{25-27}$. Therefore, it is 302 reasonable to assume that X-ray spectra are currently available.

\section{Conclusions}

305 The proposed method using the MBFPA is useful in the theoretical analysis of physical density 306 calibrations. The accuracy of SECT calibration is comparable with that of DECT calibration and is 307 improved with the use of biological tissues. The size and shape of the calibration phantom could affect 308 the accuracy, especially for MV CT, mainly because of the beam hardening effects. The present method 309 based on the MBFPA can also be applied to various other studies, such as effective atomic number 310 estimations and material decompositions. 


\section{Declarations}

\section{Ethics approval and consent to participate}

314 Not applicable.

\section{Consent for publication}

316 Not applicable.

\section{Availability of data and materials}

318 The datasets used and/or analysed during the current study are available from the corresponding author

319 upon reasonable request.

320 Competing interests

321 The authors declare that they have no competing interests.

\section{$322 \quad$ Funding}

323 This work was partially supported by JSPS KAKENHI (Grant No. 19K08201) and by the National 324 Natural Science Foundation of China (Grant No. 11735003).

\section{Authors' contributions}

$326 \mathrm{KL}$ and DF conceived the idea. The method was discussed for KL, DF, and AH. Based on the discussion, $327 \mathrm{KL}$ and $\mathrm{AH}$ developed the software, and KL and DF analysed the generated data. KL, AH, HL, and LG 328 presented the obtained results. KL and AH drafted the manuscript. All authors read, modified, and 329 approved the manuscript.

\section{Acknowledgements}

331 The authors thank Elekta for providing the structural information of the scanner heads for the MC 332 simulation of XVI systems. 


\section{Appendix: Material-based forward projection algorithm (MBFPA)}

The material-based forward projection algorithm applied in the X-ray virtual projections is briefly introduced here. According to Lambert-Beer's law, the photon number $n_{i}$ in the $i$-th detector after penetrating the object with attenuation coefficient $\mu_{j}$ in voxel $j$ is as follows:

$$
n_{i}(E)=n_{0}(E) e^{\sum_{j}-a_{i j} \mu_{j}(E)}
$$

where $E$ is the photon energy, $n_{0}$ is the photon number in the X-ray source, and $a_{i j}$ is the photon pass length in voxel $j$, representing an element of the "system matrix". If the spectrum of the X-ray is considered (as bins), the total photon number in the $i$-th detector becomes the following:

$$
n_{i}^{\text {total }}=\sum_{E} \alpha(E) n_{i}(E)=\sum_{E} \alpha(E) n_{0}(E) e^{\sum_{j}-a_{i j} \mu_{j}(E)},
$$

where $\alpha(E)$ is the fraction of the corresponding photon energy bin. $\mu_{j}(E)$ is dependent on the atomic number $Z$ and the density $\rho$ of the materials in a voxel $j$, which is expressed as a sum of the attenuation coefficients for each element $m$ as follows:

$$
\mu_{j}(E, Z, \rho)=\sum_{m} w_{m} \mu_{m, j}(E, Z, \rho)
$$

where $w_{m}$ denotes the weight (fraction) of the $m$ th element. For the energy range considered in this study, the attenuation coefficient $\mu_{m, j}(E, Z, \rho)$ can be written as the sum of the processes of the photoelectric effect, Compton scattering, and pair production as follows:

$$
\mu_{m, j}(E, Z, \rho)=\rho Z \frac{N_{A}}{A}\left[\sigma_{p e}(E, Z)+\sigma_{c o m p}(E)+\sigma_{p p}(E, Z)\right]
$$

where $N_{A}$ and $A$ denote the Avogadro constant and atomic weight, respectively. $\sigma$ is the cross section of the photon - matter interactions. The cross section of the photoelectric effect can be approximated by ${ }^{28}$ as follows:

$$
\sigma_{p e}(E, Z)=3.45 \times 10^{-6} r_{e}^{2}(1+0.008 Z) \frac{Z^{3}}{E^{3}}\left(1-\frac{E_{k}}{4 E}-\frac{E_{k}^{2}}{1.21 E}\right)
$$

where $r_{e}=2.81794 \mathrm{fm}$ is the classical electron radius and $E_{k}$ is the $K$-shell binding energy. The latter is ignored in this study. The Compton scattering cross section is theoretically expressed by the Klein-Nishina formula as follows: 


$$
\sigma_{\text {comp }}(E)=2 \pi r_{e}^{2}\left\{\frac{1+E}{E^{2}}\left[\frac{2(1+E)}{1+2 E}-\frac{\ln (1+2 E)}{E}\right]+\frac{\ln (1+2 E)}{2 E}-\frac{1+3 E}{(1+2 E)^{2}}\right\} .
$$

359 The cross section of the pair production is approximated $\operatorname{as}^{28}$ follows:

$$
\sigma_{p p}(E, Z)=0.2545 r_{e}^{2}(E-2.332) \frac{Z}{137} .
$$

361 As a result, the virtual projections were simulated by a ray-tracing method to generate sinograms. The sinograms were simulated by considering the energy spectrum with a bin width of $1 \mathrm{keV}$ in this study. 363 


\section{References}

1. Mull RT. Mass estimates by computed tomography: physical density from CT numbers. Am J Roentgenol 1984;143(5):1101-1104.

2. Langen KM, Meeks SL, Poole DO, Wagner TH, Willoughby TR, Kupelian PA, Ruchala KJ, Haimerl J, Olivera GH. The use of megavoltage CT (MVCT) images for dose recomputations. Phys Med Biol 2005;50(18):4259.

3. Yang Y, Schreibmann E, Li T, Wang C, Xing L. Evaluation of on-board kV cone beam CT (CBCT)-based dose calculation. Phys Med Biol 2007;52(3):685.

4. Verhaegen F, Devic S. Sensitivity study for CT image use in Monte Carlo treatment planning. Physics in Medicine \& Biology, 50:937, 2005.

5. Fang R, Mazur T, Mutic S, Khan R, The impact of mass density variations on an electron Monte Carlo algorithm for radiotherapy dose calculations. Phys Imag Radiat Oncol 2018;8:1.

6. Cozzi L, Fogliata A, Buffa F, Bieri S. Dosimetric impact of computed tomography calibration on a commercial treatment planning system for external radiation therapy. Radiother Oncol $1998 ; 48(3): 335-338$.

7. Schaffner B, Pedroni E. The precision of proton range calculations in proton radiotherapy treatment planning: experimental verification of the relation between CT-HU and proton stopping power. Phys Med Biol 1998;43(6):1579.

8. Schneider U, Pedroni E, Lomax A. The calibration of CT Hounsfield units for radiotherapy treatment planning. Phys Med Biol 1996;41(1):111.

9. Gomá C, Almeida IP, Verhaegen F. Revisiting the single-energy CT calibration for proton therapy treatment planning: a critical look at the stoichiometric method. Phys Med Biol 2018;63(23):235011.

10. Michalak G, Taasti V, Krauss B, Deisher A, Halaweish A, McCollough C. A comparison of relative proton stopping power measurements across patient size using dual- and single-energy CT. Acta Oncol 2017;56:1465-71. 
11. Yang M, Virshup G, Clayton J, Zhu XR, Mohan R, Dong L. Theoretical variance analysis of single-and dual-energy computed tomography methods for calculating proton stopping power ratios of biological tissues. Phys Med Biol 2010;55(5):1343.

12. Hunemohr N, Paganetti H, Greilich S, Jakel O, Seco J. Tissue decomposition from dual energy ct data for mc based dose calculation in particle therapy. Med Phys 2014;41(6Part1):061714.

13. II ICRU. Tissue substitutes in radiation dosimetry and measurement. International Commission on Radiation Units and Measurements, 1989.

14. White DR, Woodard HQ, Hammond SM. Average soft-tissue and bone models for use in radiation dosimetry. Brit J Radiol 1987;60(717):907-913.

15. Woodard HQ, White DR. The composition of body tissues. Brit J Radiol 1986;59(708):12091218.

16. Elbakri, IA, Fessler JA. Statistical image reconstruction for polyenergetic x-ray computed tomography. IEEE Trans Med Imaging 2002;21(2):89-99.

17. Shen L, Xing Y. Multienergy CT acquisition and reconstruction with a stepped tube potential scan. Med Phys 2015;42(1):282-296.

18. Ruchala KJ, Olivera GH, Schloesser EA, Mackie TR: Megavoltage CT on a tomotherapy system. Phys Med Biol 1999;44:2597-2621.

19. Wertz H, Stsepankou D, Blessing M, Rossi M, Knox C, Brown K, Gros U, Boda-Heggemann J, Walter C, Hesser C, Lohr F, Wenz F: Fast kilovoltage/megavoltage (kVMV) breathhold conebeam CT for image-guided radiotherapy of lung cancer, Phys Med Biol 2010;55:4203-4217.

20. Pouliot J, Bani-Hashemi A, Chen J, et al. Low-dose megavoltage cone-beam CT for radiation therapy. Int J Radiat Oncol Biol Phys 2005;61:552-560.

21. Sakata D, Haga A, Kida S, Imae T, Takenaka S, Nakagawa K. Effective atomic number estimation using kV-MV dual-energy source in linac. Phys Medica, 2017;39:9-15.

22. Saito M. Potential of dual-energy subtraction for converting CT numbers to electron density based on a single linear relationship. Med Phys 2012;39.

23. Tsukihara M, Noto Y, Sasamoto R, Hayakawa T, Saito M. Initial implementation of the conversion from the energy-subtracted CT number to electron density in tissue inhomogeneity 

$2015 ; 42$.

24. Yang M, Virshup G, Clayton J, Zhu XR, Mohan R, Dong L. Does kV-MV dual-energy computed tomography have an advantage in determining proton stopping power ratios in patients? Phys Med Biol 2011;56(14):4499.

25. Sidky EY, Yu L, Pan X, Zou Y, Vannier M. A robust method of x-ray source spectrum estimation from transmission measurements: Demonstrated on computer simulated, scatter-free transmission data. J Appl Phys 2005;97(12):124701.

26. Duan X, Wang J, Yu L, Leng S, McCollough CH. CT scanner X-ray spectrum estimation from transmission measurements. Med Phys 2011;38(2):993-997.

27. Ha W, Sidky EY, Barber RF, Schmidt TG, Pan X. Estimating the spectrum in computed tomography via Kullback-Leibler divergence constrained optimization. Med Phys 2019;46(1):8192.

28. Yao W, Leszczynski KW. An analytical approach to estimating the first order x-ray scatter in heterogeneous medium. Med Phys 200936(7):3145-3156. 


\section{Figures}

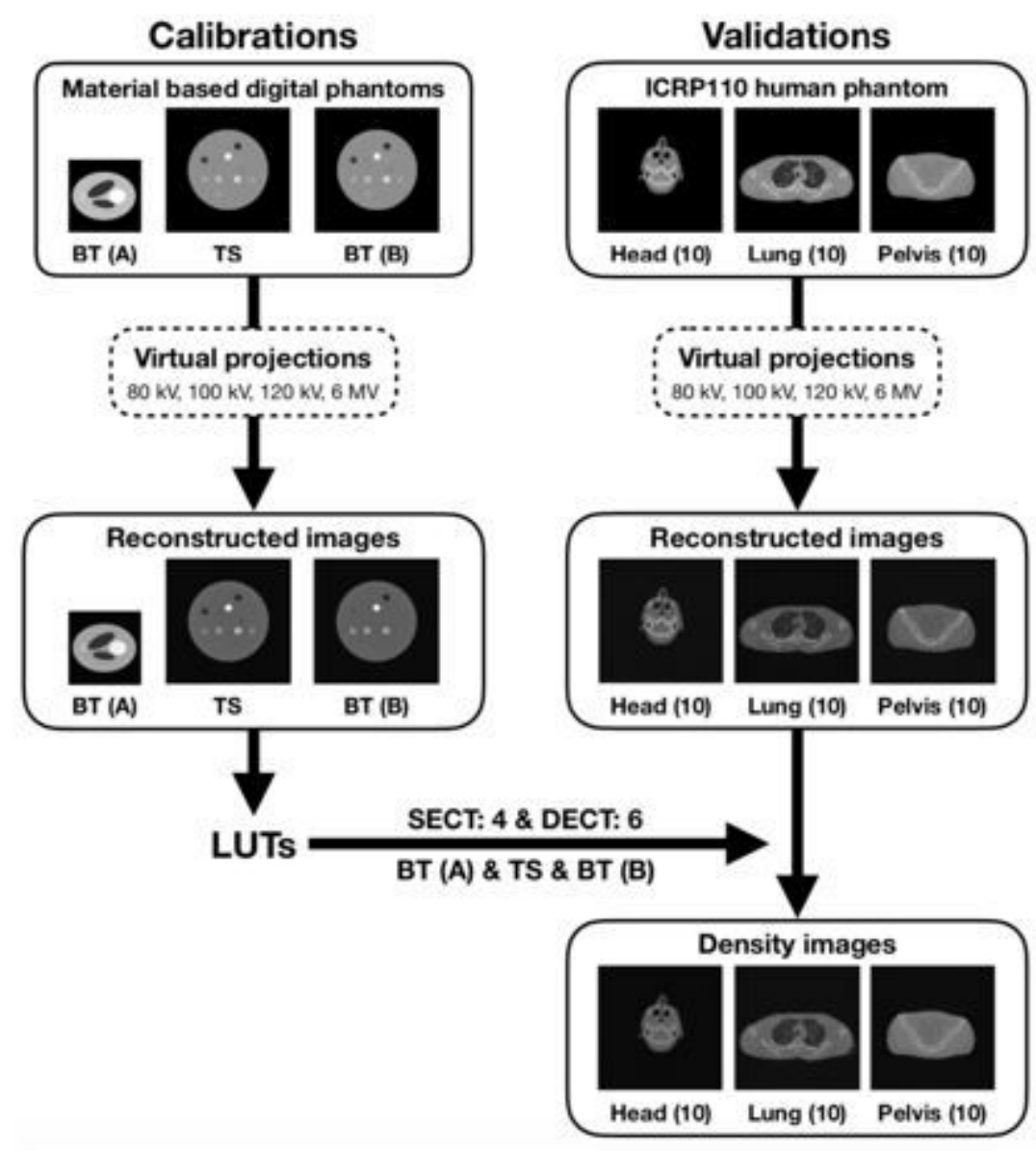

\section{Figure 1}

Workflow of the current study for total density evaluation. BT(A), TS, and BT(B) phantoms were used for the calibration, and the ICRU human phantoms were used for the validation (for more details, see the main text). 


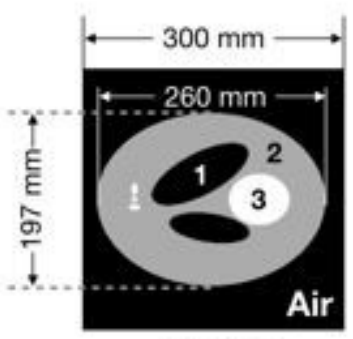

BT(A)

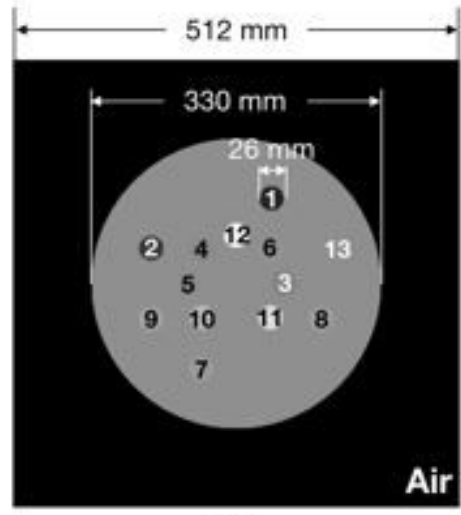

TS

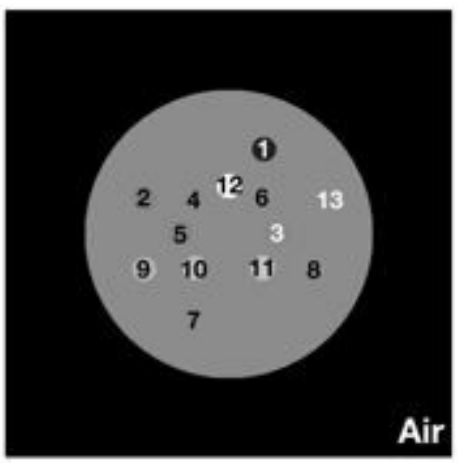

BT(B)

- BT(A) 1-3: biological tissues

- TS 1: LN-300, 2: LN-450, 3: AP6, 4: BR12, 5: Water, 6: SR2, 7: LV1, 8: IB3, 9: B200, 10: CB2-30\%CaCO3, 11: CB2-50\%CaCO3, 12: SB3, 13: Solid water

- BT(B) 5: Water, Others: biological tissues

Figure 2

Shape, size, and composition of the BT(A), TS, and BT(B) phantoms in the calibration.
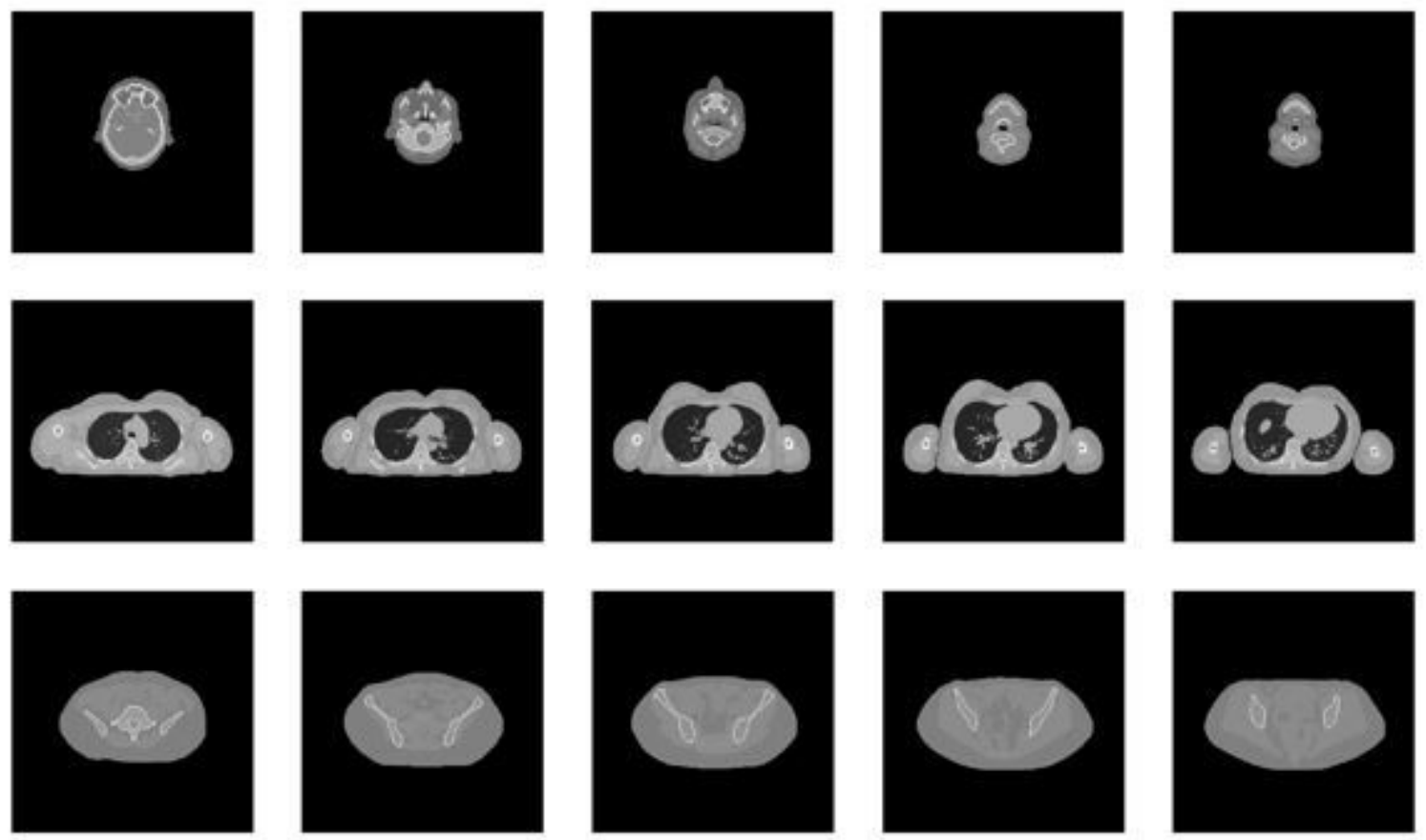

\section{Figure 3}

Ground truth of the selected ICRP110 human phantoms for female. The images in the first, second, and third row indicate head, lung, and pelvis, respectively. 

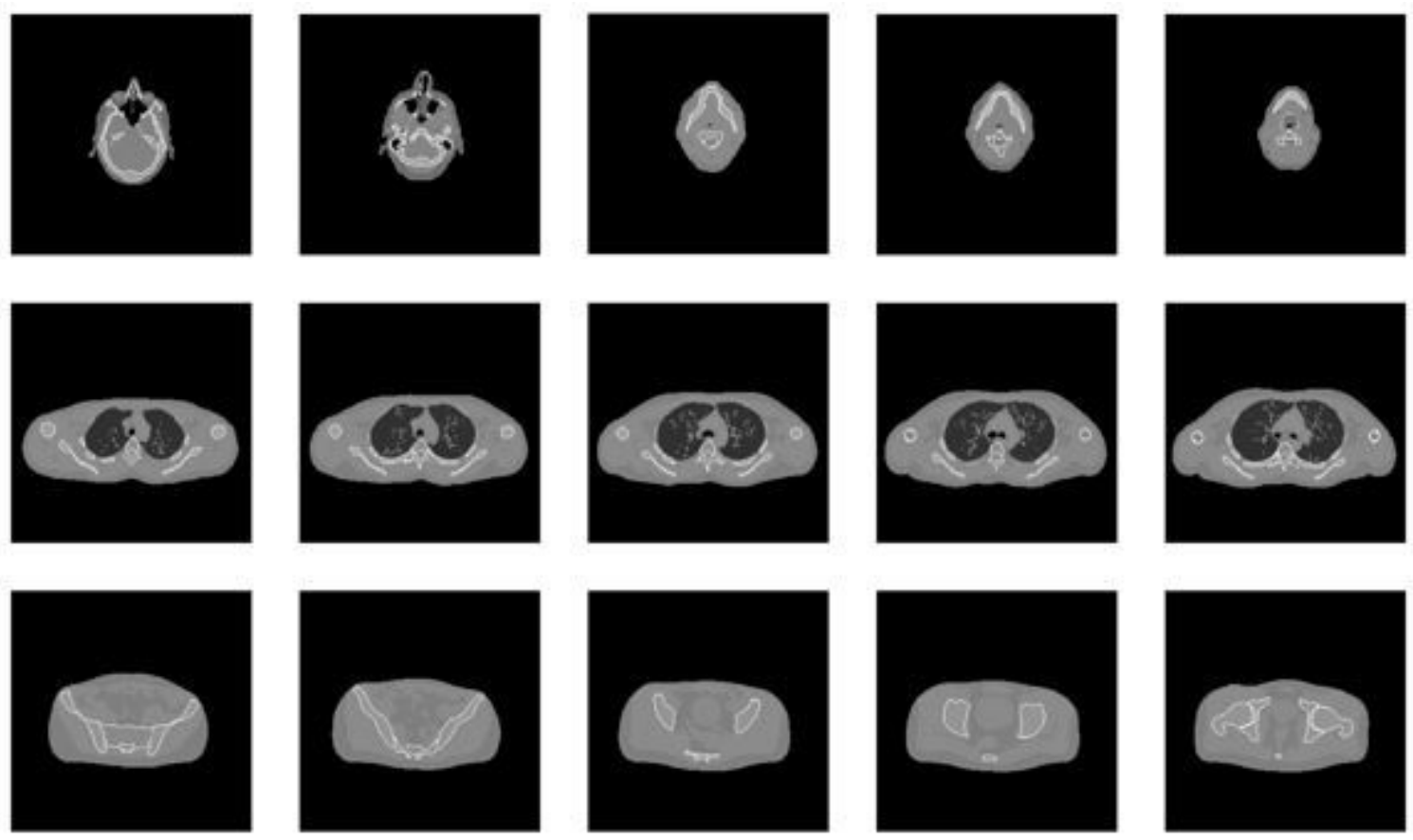

Figure 4

Ground truth of the selected ICRP110 human phantoms for male. The images in the first, second, and third row indicate head, lung, and pelvis, respectively.
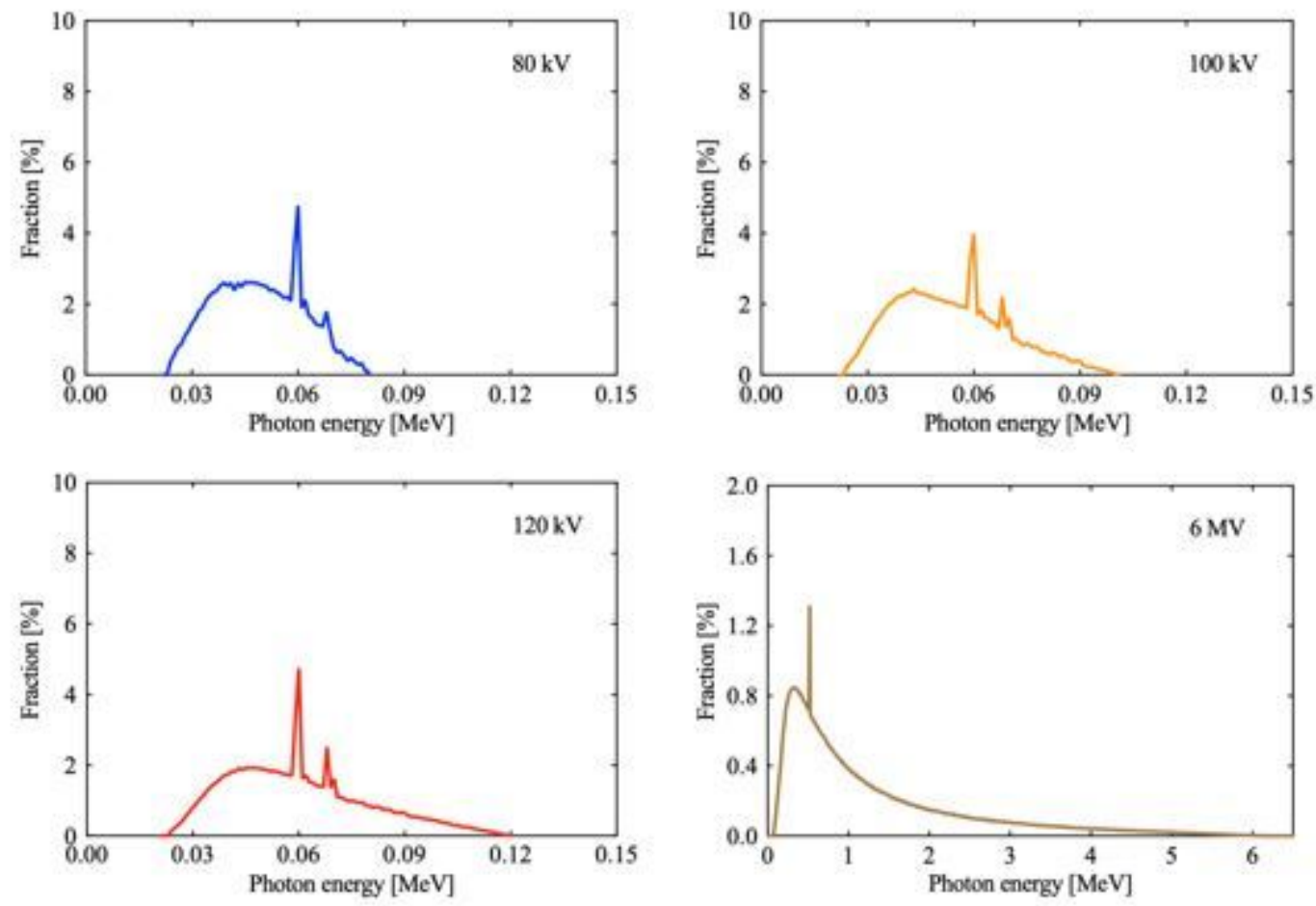

Figure 5

Simulated X-ray spectra of $80 \mathrm{kV}, 100 \mathrm{kV}, 120 \mathrm{kV}$, and $6 \mathrm{MV}$. The mean energies were $48.70 \mathrm{keV}, 53.24 \mathrm{keV}$, $59.28 \mathrm{keV}$, and $1.30 \mathrm{MeV}$, respectively. 


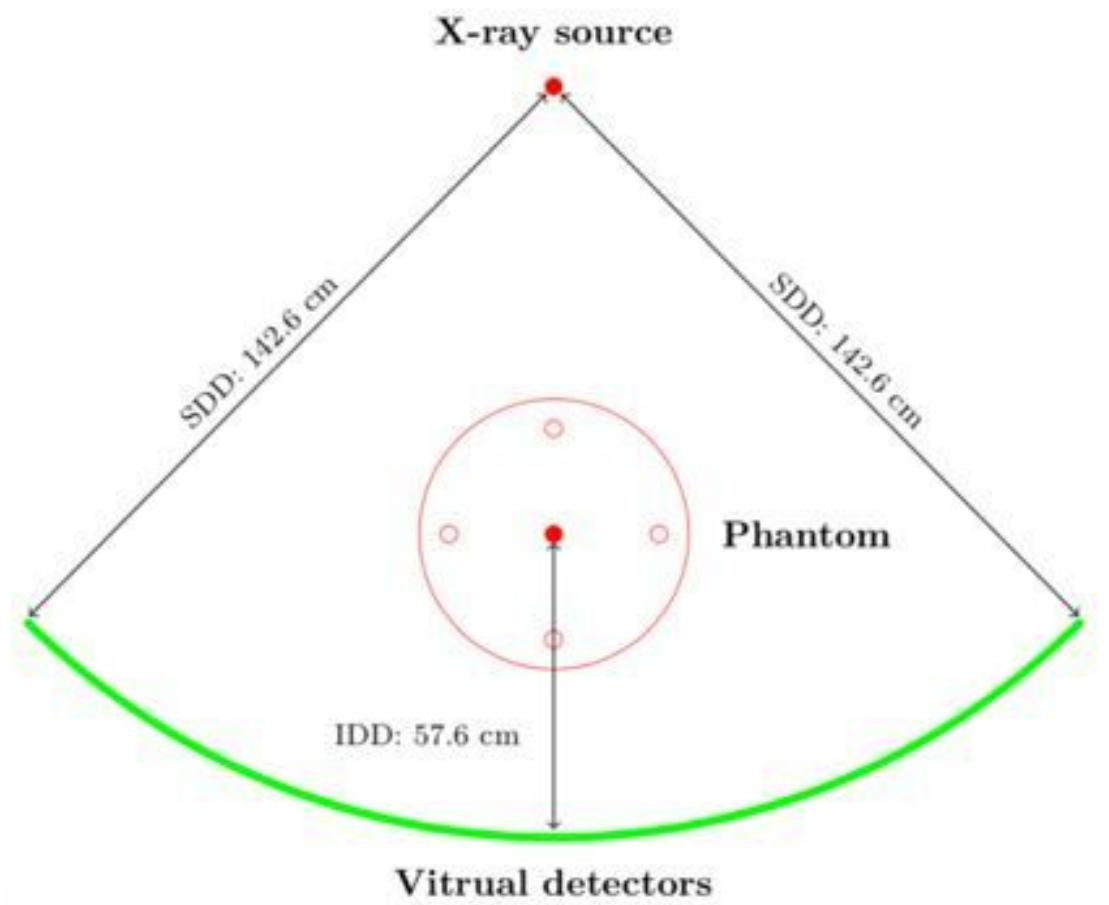

Figure 6

Schematic of geometry applied in sinogram production. IDD and SDD indicate the isocenter-to-detector distance and the source-to-detector distance, respectively. A total of 609 detectors were used, and the size of each one was $0.15 \mathrm{~cm}$.
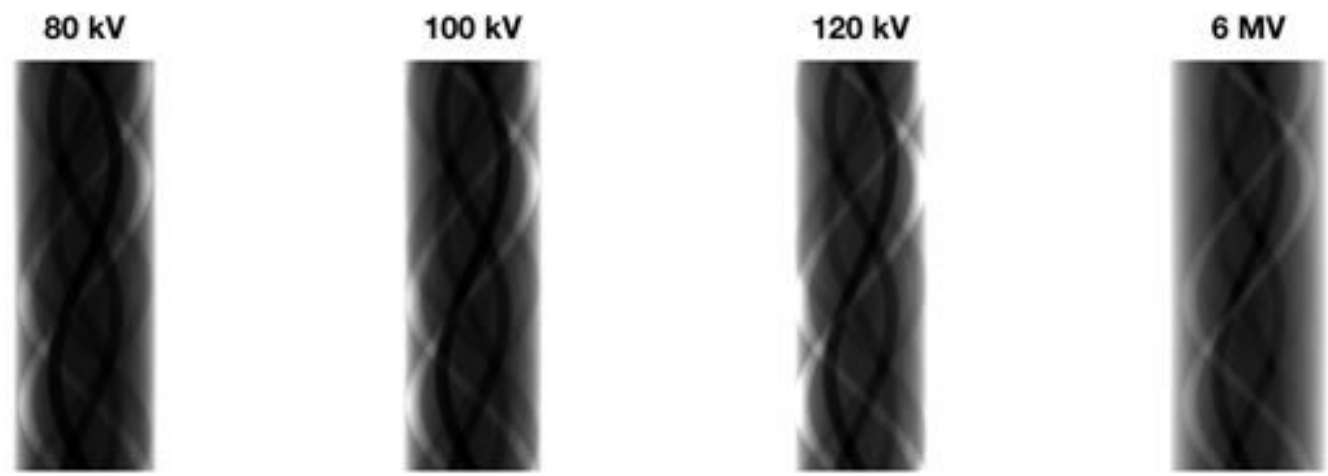

\section{Figure 7}

Sinograms of the TS phantom with $80 \mathrm{kV}, 100 \mathrm{kV}, 120 \mathrm{kV}$, and $6 \mathrm{MV}$. The display value ranges are $0-0.01$ for $\mathrm{kV}$ CT and 0.1-0.3 for MV CT. 

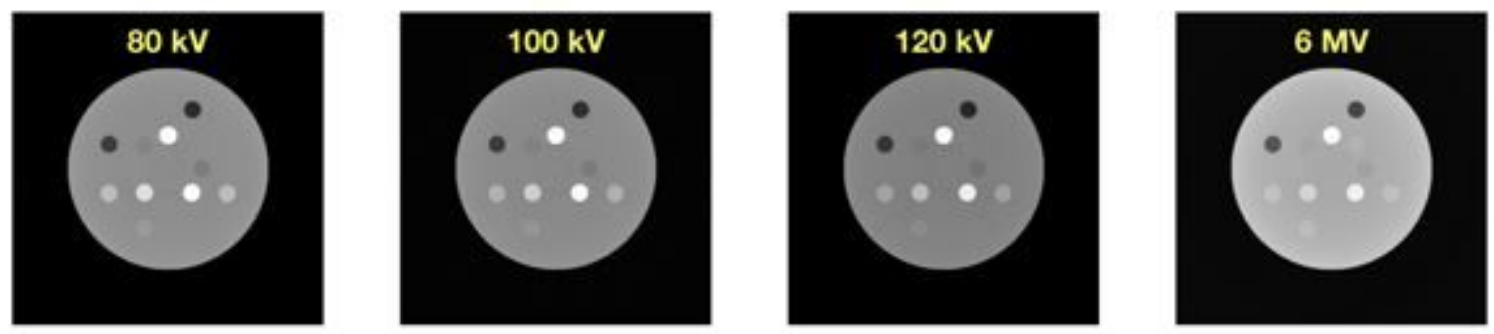

Figure 8

Reconstructed images of the TS phantom with $80 \mathrm{kV}, 100 \mathrm{kV}, 120 \mathrm{kV}$, and $6 \mathrm{MV}$. The display value ranges of the attenuation coefficients (in units of $\mathrm{cm}-1$ ) are $0-0.4$ for $\mathrm{kV}$ CT and $0-0.1$ for MV CT.
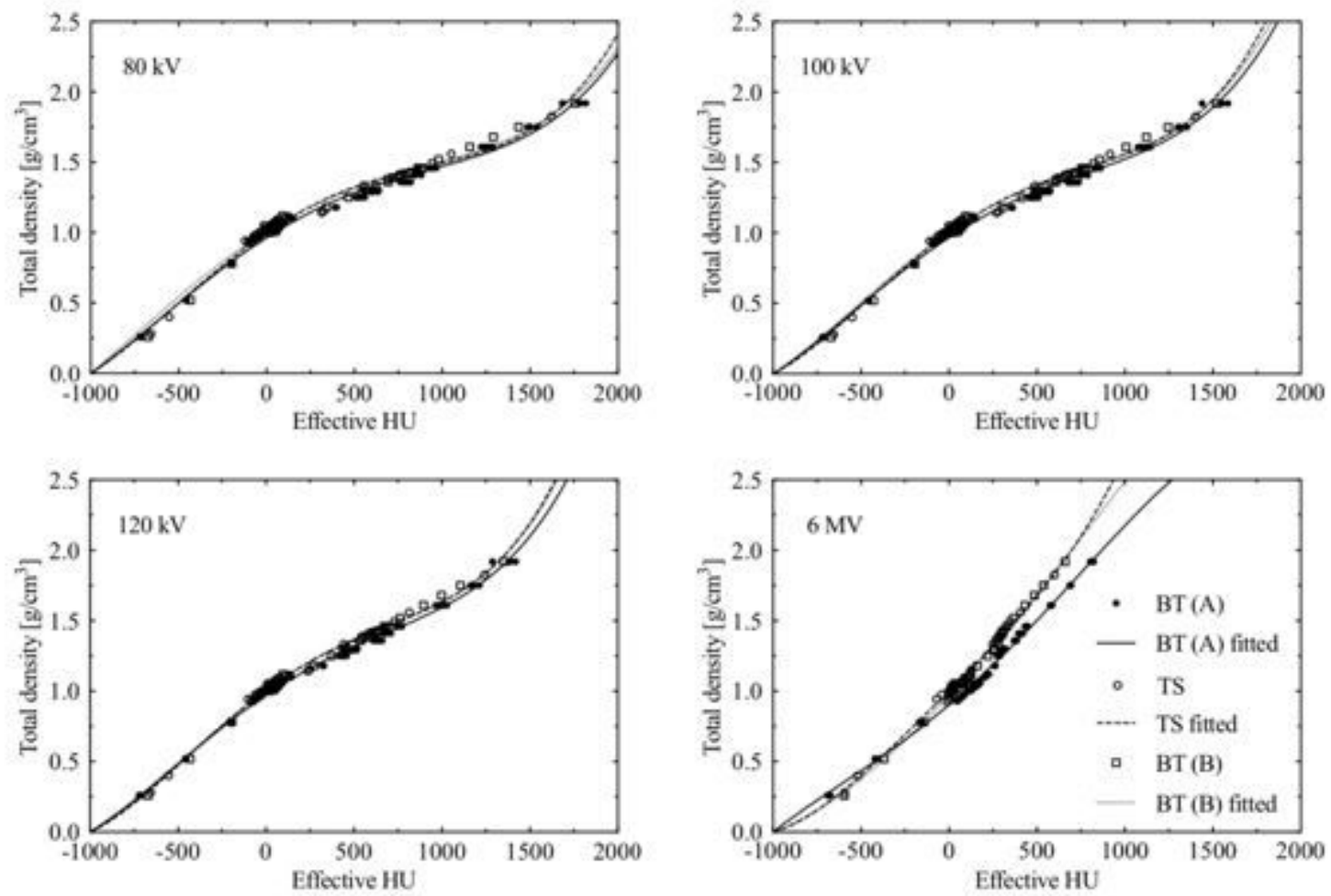

\section{Figure 9}

HU-to-density relations of three calibration phantoms with $80 \mathrm{kV}, 100 \mathrm{kV}, 120 \mathrm{kV}$, and $6 \mathrm{MV}$ in SECT. The CT values are referred to as "Effective $\mathrm{HU}$ " because this approach is based on simulations. 

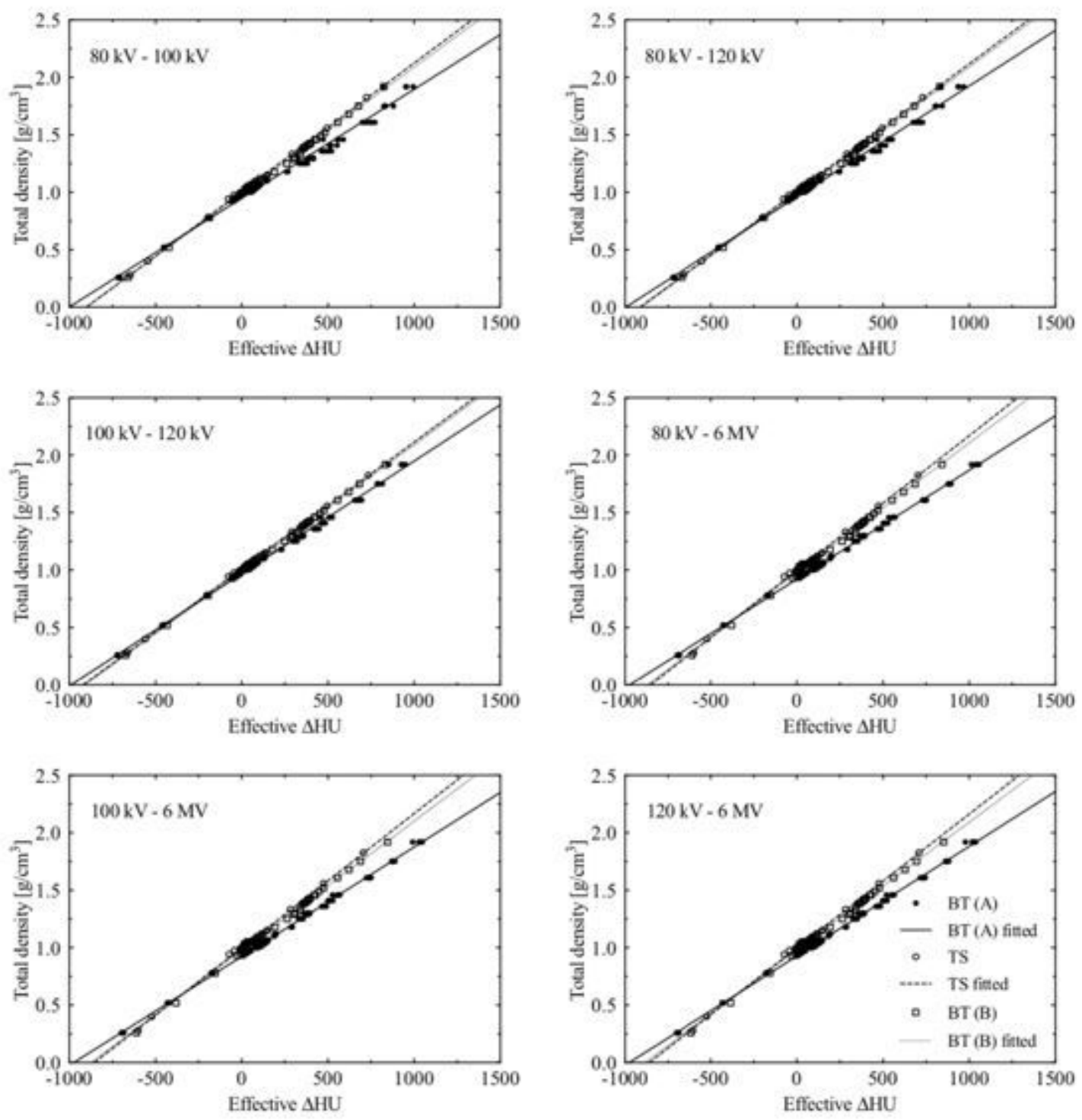

Figure 10

Effective $\Delta \mathrm{HU}$ to density relations of the three calibration phantoms with $80 \mathrm{kV}, 100 \mathrm{kV}, 120 \mathrm{kV}$, and $6 \mathrm{MV}$ in DECT. 